\title{
Encapsulation of Quercetin in Tri-Block- Copolymer/Tween 80 Mixed Nanomicelles to Enhance its Cytotoxicity Against Breast Cancer Cells
}

\section{Reza Davarnejad}

Arak University

Kiyana Layeghy

Arak University

Meysam Soleymani ( $\square$ m-soleymani-chem@araku.ac.ir)

Arak University https://orcid.org/0000-0003-1585-5880

Arvin Ayazi

Arak University

\section{Research Article}

Keywords: Breast cancer, Pluronic F127, Quercetin, Mixed micelles, Drug delivery

Posted Date: November 16th, 2021

DOI: https://doi.org/10.21203/rs.3.rs-1075658/v1

License: (c) (1) This work is licensed under a Creative Commons Attribution 4.0 International License.

Read Full License 


\section{Abstract}

Quercetin, a natural polyphenolic compound, has attracted much attention due to its great therapeutic potential against various types of diseases. But clinical applications of quercetin are limited due to its poor aqueous solubility and low bioavailability. The main purpose of this research was to evaluate the therapeutic potential of quercetin-loaded Pluronic F127 (PF127)/Tween 80 mixed nanomicelles as a passive targeted drug delivery system for breast cancer therapy. To this end, quercetin-loaded mixed nanomicelles with different mass ratios of drug:PF127:Tween 80 were prepared by the thin-film hydration method. The highest drug loading and entrapment efficiency were obtained to be $2.3 \%$ and $98.0 \%$, respectively, for mixed micelles with drug:PF127:Tween 80 ratio of 1:40:15. The physical interactions of quercetin with PF127 and Tween 80 at optimized ratio was investigated by XRD and FTIR analyses. The mean hydrodynamic size and surface charge of prepared nanomicelles, measured by DLS and zeta potential analyses, were $22.1 \mathrm{~nm}$ and $-7.63 \mathrm{mV}$, respectively. The results of in-vitro drug release experiments showed that, the mixed micellar system has a prolong and sustained release behavior compared to the solution of free quercetin. Moreover, the in-vitro cytotoxicity studies of quercetin-loaded mixed nanomicelles on breast cancer cells (MCF-7) revealed that, the encapsulated drug have a lower $\mathrm{IC}_{50}$ value $(8.9 \mu \mathrm{g} / \mathrm{mL})$ compared to the free drug $(49.2 \mu \mathrm{g} / \mathrm{mL})$. Our results suggest that, quercetinloaded mixed nanomicelles can be considered as a promising drug delivery system with prolonged release and potentiated cytotoxicity against breast cancer cells.

\section{Introduction}

Quercetin, a polyphenolic flavonoid present in many fruits, vegetables, and grains has extensively received wide attention due to its wide range of pharmaceutical properties including wound healing, antiinflammatory, antioxidant, antibacterial, and hepatoprotective [1-5]. Many studies have shown that, the potential therapeutic of quercetin on different kinds of cancers such as hepatocellular carcinoma [6], breast cancer [7], prostate cancer [8], and lung cancer [9]. Moreover, the synergistic effects of co-delivery of quercetin with other anticancer drugs on the therapy of the invasive cancers have been investigated $[10,11]$. Despite such a wide range of medicinal properties, clinical applications of quercetin are limited due to its poor aqueous solubility which results in short biological half-life, and low bioavailability of drug. One of the best ways to enhance the water solubility and chemical stability of quercetin is its encapsulation into polymeric micelles [12]. Generally, encapsulation of hydrophobic drugs by polymeric micelles has been preferred as an efficient drug delivery system against many diseases [13-17]. Polymeric micelles composed of poloxamer (Pluronic) tri-block copolymers with average particle size less than $100 \mathrm{~nm}$ exhibit many advantages including encapsulation of hydrophobic drugs, long systemic circulation time, and targeting ability [18-22]. In aqueous solutions and at concentrations above critical micelle concentration (CMC), these tri-block copolymers self-assemble into spherical or cylindrical micelles with a hydrophobic core composed of polypropylene oxide (PPO) blocks and hydrophilic shell due to the polyethylene oxide (PEO) chains $[23,24]$. The hydrophobic cores of formed micelles can be used as a suitable space for the encapsulation of hydrophobic compounds such as hydrophobic drugs, 
while the hydrophilic chains are responsible to keep the micelles stable and decrease undesirable drug interactions with outer medium [25]. Therefore, the encapsulation of drugs using Pluronic micelles leads to increasing solubility, bioavailability, and blood circulation time [26]. US Food and Drug Administration (FDA) have approved various types of pluronic copolymers for pharmaceutical applications including Pluronic F127 (PF127) $[27,28]$. Up to now, many research have been carried out to improve the solubility, stability, and efficacy of different hydrophobic drugs used in cancer treatment by PF127 micelles [21, 29]. However, micellar systems formed by only PF127 exhibit low drug loading and low entrapment efficiency [30]. Hence, binary micellar systems containing different kinds of Pluronics, or combination of Pluronic with amphiphilic molecules, anionic or cationic surfactants have been proposed [19, 31-34]. Tween 80 (Polysorbate 80 ), is a biocompatible nonionic surfactant which can improve aqueous solubility of hydrophobic drugs through micelles formation. Furthermore, Tween 80 can enhance the permeability of different drugs across biological membranes [35].

In the present work, a mixed micellar system composed of PF127 and Tween 80 is proposed for enhancing the loading content and entrapment efficiency of quercetin in a mixed micellar system, which has not been reported so far. Quercetin-loaded mixed micelles were synthesized via thin-film hydration method and then characterized in terms of drug loading, entrapment efficiency, morphology, particle size and surface properties. Also, the in vitro drug release of quercetin from PF127/Tween 80 mixed nanomicelles was compared to free drug at PBS solution ( $\mathrm{pH} 7.4)$. Finally, in vitro cytotoxic activity of quercetin-loaded mixed micelles was investigated against MCF7 cancer cells.

\section{Experimental}

\subsection{Materials}

Pluronic ${ }^{\circledR}$ F-127 ( $\left.\mathrm{PEO}_{100}-\mathrm{PPO}_{65}-\mathrm{PEO}_{100}, \mathrm{M}_{\mathrm{w}}=12,600 \mathrm{~g} / \mathrm{mol}\right)$, Quercetin ( $\geq 98 \%, \mathrm{HPLC}$ ), High retention seamless cellulose tubing (dialysis tubing, $99.99 \%$ retention) with a molecular weight cut-off (MWCO) $12400 \mathrm{Da}$, and Phosphate buffer solution (PBS, 1.0 M, pH 7.4 $\left(25^{\circ} \mathrm{C}\right)$ ) were purchased from SigmaAldrich Company (Steinheim, Germany). Methanol (99.9\%), and dimethyl sulfoxide (DMSO, $\geq 99.9 \% \mathrm{GC}$ ) were prepared from Merck company (Darmstadt, Germany). Tween 80 was purchased from Daejung company (Siheung, Korea). Deionized water was used for all aqueous preparations.

\subsection{Quercetin-loaded mixed micelles}

Quercetin-loaded mixed micellar systems were prepared via thin-film hydration method $[36,37]$. To this end, different mass ratios of quercetin:PF127:Tween 80 (1:40:0, 1:40:5, 1:40:10, and 1:40:15) were dissolved in $30 \mathrm{~mL}$ of methanol in a round-bottom flask. Subsequently, the solvent was evaporated at 50 ${ }^{\circ} \mathrm{C}$ overnight to obtain a uniform thin film. Deionized water was then added to the film along with stirring at $600 \mathrm{rpm}$ for $1 \mathrm{~h}$ to form a clear greenish micellar solution. The solution was then filtrated through 0.22 $\mu \mathrm{m}$ filter to eliminate the unencapsulated quercetin.

\subsection{Characterizations}


The physical interactions of quercetin with mixed micelles were studied by Fourier Transform Infrared Spectroscopy (FTIR, Alpha II, Bruker Optik GmbH, Ettlingen, Germany), and X-ray Powder Diffraction (XRD) analysis (Malvern Panalytical X' Pert Pro, UK, Cr Ka radiation). The hydrodynamic size distribution and surface charge of the nanomicelles were measured by dynamic light scattering and zeta potential analyses (DLS, Malvern, Zetasizer, UK). The concentration of quercetin in drug loading and release studies was determined by UV-Vis Spectrophotometer (PerkinElmer, Lambda 25, USA) at the wavelengths of $374 \mathrm{~nm}$ for quercetin. The UV-Vis spectrophotometer was calibrated with standard solutions of 1 to 25 $\mu \mathrm{g} / \mathrm{mL}$ of quercetin dissolved in ethanol $\left(R^{2}=0.99\right)$.

\subsection{In vitro drug release experiments}

After encapsulation of quercetin in mixed micelles, the concentration of drug incorporated drug in micellar system was determined by UV-vis spectroscopy. For this purpose, a certain volume of micellar solution was diluted with certain amount of ethanol to disrupt the micelles, and then the concentration of quercetin was determined from the calibration curve obtained for pure quercetin. The drug-loading (DL\%) and entrapment efficiency (EE\%) of the quercetin were determined according to the following equations [38]:

$$
\begin{aligned}
& E E \%=\frac{\text { Weight of drug encapsulated in micelles }}{\text { Weight of drug initially added }} \times 100 \\
& D L \%=\frac{\text { Weight of drug encapsulated in micelles }}{\text { Weight of drug-loaded mixed micelles }} \times 100
\end{aligned}
$$

The release of quercetin as a free drug and encapsulated in PF127/Tween 80 mixed micelles was performed at PBS solution $\left(\mathrm{pH}=7.4\right.$ and $\left.37^{\circ} \mathrm{C}\right)$ to simulate the in vivo physiological environment. To this end, a certain amount of freeze-dried quercetin-loaded mixed micelles was dispersed in $5 \mathrm{~mL}$ PBS and inserted into a dialysis bag (MWCO 12000) and then immersed into $50 \mathrm{~mL}$ of PBS solution $(0.1 \%$ tween $\mathrm{v}: \mathrm{v})$. At different intervals, $1 \mathrm{~mL}$ of release medium was withdrawn and replaced with an equal volume of fresh medium. The concentration of quercetin in each sample was determined by UV-vis spectrophotometry at $374 \mathrm{~nm}$ after dilution with certain amount of ethanol. The cumulative drug release percentage $(Q \%)$ was calculated according to the following equation [38]:

$$
Q \%=\frac{\text { Weight of quercetin released in PBS buffer solution }}{\text { Weight of quercetin in the dialysis bag }} \times 100
$$

\subsection{In vitro cytotoxicity analysis}

The cytotoxicity of free quercetin, blank mixed micelles and quercetin-loaded mixed micelles on human breast cancer cells (MCF7) was evaluated using 3-(4,5-dimethylthiazol-2-yl)-2,5diphenyltetrazolium bromide (MTT) assay. To perform this assay, cells were seeded in a 48-well plate at 
density of about $2 \times 10^{4}$ cells/well and incubated overnight to allow cells attachment. After incubation, the medium of each well was replaced with fresh medium containing free quercetin ( $0.5 \% \mathrm{v}: \mathrm{v}$ DMSO) or equivalent doses of drug in mixed nanomicelles with concentrations of $0,0.5,5,25$, and 50 $\mu \mathrm{g}_{\text {quercetin }} / \mathrm{mL}$. After $48 \mathrm{~h}$ incubation, the medium of each well was removed, the wells were rinsed with PBS. Afterwards, $50 \mu \mathrm{L}$ of MTT solution $(2 \mathrm{mg} / \mathrm{mL})$ was added to each well followed by $4 \mathrm{~h}$ incubation. The medium was discarded and $200 \mu \mathrm{l}$ of DMSO was added to each well to solubilized the formazan crystal. Relative cell viability of each group compared to control group was determined by measuring the optical absorbance at $570 \mathrm{~nm}$

\subsection{Data analysis}

All in vitro experiments were performed in triplicate, and GraphPad Prism 6 software was used for data processing. Difference between mean values of each pair was considered for significance using one-way analysis of variance (ANOVA) and Student's $t$-test.

\section{Results And Discussion}

Polymeric micelles have been extensively studied as anticancer drug delivery carriers in preclinical experiments and some nano-dimensional drug delivery systems based on these formulations are currently undergoing clinical trials [12]. Generally, Pluronic micelles containing anticancer drugs, have shown prolonged half-life in comparison to free drug, and also higher efficacy in treatment of patients with advanced cancer compared to standard protocol of chemotherapy [12, 39]. In this study, to optimize the composition of mixed nanomicelles, changes in the drug loading and encapsulation efficiency were measured as a function of the drug:PF127:Tween 80 (w:w:w) ratio. It should be noted that, when the drug:PF127 (w/w) ratio was less than 1:40 (single nanomicelles), the quercetin precipitated after several days, indicating the unstable encapsulation of quercetin in the hydrophobic core of prepared nanomicelles. Therefore, the ratio of drug:PF127 was fixed as 1:40 for the formation of the mixed nanomicelles. In the prepared nanomicelles, the content of Tween 80 affected on the entrapment efficiency and drug loading of quercetin in the mixed nanomicelles and results of different contents of Tween are presented in Table 1. As can be observed, the drug loading and entrapment efficiency of the nanomicelles increased with increasing the Tween 80 content. The entrapment efficacy and drug loading of quercetin into the mixed nanomicelles was reached to $98 \%$, and $2,3 \%$, respectively, with drug:PF127:Tween 80 ratio of 1:40:15. So, this composition was used for continued study. 
Table 1

Drug loading and entrapment efficiency for mixed nanomicelles with different compositions

\begin{tabular}{|lll|}
\hline Quercetin:PF127:Tween (w:w:w) & Drug Loading (\%) & Entrapment Efficiency (\%) \\
\hline $1: 40: 0$ & 1.90 & 75.0 \\
\hline $1: 40: 5$ & 2.10 & 86.2 \\
\hline $1: 40: 10$ & 2.23 & 91.8 \\
\hline $1: 40: 15$ & 2.30 & 98.0 \\
\hline
\end{tabular}

FT-IR spectra of free quercetin, empty mixed nanomicelles, and quercetin-loaded mixed nanomicelles are presented in Fig. 1. FTIR spectrum of pure quercetin is shown in Fig. 1a. The broad absorption band appeared at $3100-3500 \mathrm{~cm}^{-1}$ is attributed to the stretching vibrations of hydroxyl $(-\mathrm{OH})$ groups. The band at $1664 \mathrm{~cm}^{-1}$ is related to the stretching of $\mathrm{C}=0$ bond in quercetin molecule. The absorption bands at 1609,1561 , and $1520 \mathrm{~cm}^{-1}$ can be assigned to the stretching vibrations of $\mathrm{C}=\mathrm{C}$ bonds in aromatic rings [40]. The bands detected at 1261, 1199, and $1168 \mathrm{~cm}^{-1}$ are related to the stretching vibrations of $\mathrm{C}-0$ bonds in the ether ring, the stretching of $\mathrm{C}-\mathrm{O}$ bonds in phenol rings, and stretching and bending vibrations of C-CO-C bond in ketone, respectively [40]. Moreover, the absorption bands at 1320 and 939 $\mathrm{cm}^{-1}$ are related to the in-plane and out-of-plane bending vibrations of $\mathrm{C}-\mathrm{H}$ bonds in aromatic rings. In the FTIR spectrum of empty mixed nanomicelles (Fig. 1b), the absorption peaks at 3447, 2880, and 1242 $\mathrm{cm}^{-1}$ are attributed to the stretching vibrations of hydroxyl, alkyl $\left(-\mathrm{CH}_{2}\right)$, and $\mathrm{C}-\mathrm{C}$ bonds, respectively, in pluronic and Tween 80 molecules. The absorption band at $1735 \mathrm{~cm}^{-1}$ is due to the stretching vibration of $\mathrm{C}=\mathrm{O}$ bond in the ester carbonyl group of Tween 80 molecules [41]. Moreover, the spectrum of empty mixed nanomicelles showed two strong absorption peaks at 962 , and $1112 \mathrm{~cm}^{-1}$, which can be assigned to the asymmetric and symmetric stretching vibration of $\mathrm{C}-\mathrm{O}$ bonds in pluronic molecules. Moreover, the absorption band appeared at $1281 \mathrm{~cm}^{-1}$ is related to the stretching vibrations of $\mathrm{C}-\mathrm{O}-\mathrm{C}$ bonds in pluronic molecules. To identify the interaction between the quercetin and the mixed nanomicelles, the FTIR spectrum of quercetin-loaded mixed nanomicelles is shown in Fig. 1c. As can be observed in this Figure, the spectrum of quercetin-loaded PF127/Tween 80 micelles is similar to that of empty mixed nanomicelles and does not show characteristic peaks of quercetin. These result suggests that the quercetin molecules are located in the inner hydrophobic core of mixed nanomicelles.

This result was further confirmed by XRD analysis. The XRD graphs of pure quercetin, empty mixed nanomicelles, and quercetin-loaded PF127/Tween 80 mixed nanomicelles are shown in Fig. 2. As can be observed, XRD pattern of pure quercetin (Fig. 2a) exhibits numerous distinct peaks located at $2 \theta=16.0$, $18.5,23.8,24.1,35.8,36.6$, and $41.2^{\circ}$, indicating that the compound had high crystallinity. XRD pattern of empty mixed nanomicelles (Fig. 2b) showed two intensive peaks at 28.6 and $35.1^{\circ}$, indicating highly crystalline structure of copolymer. However, the characteristic diffraction peaks of quercetin were lost after encapsulation in mixed nanomicelles (Fig. 2c), indicating that quercetin as an amorphous or 
disordered crystalline phase has successfully been incorporated into the hydrophobic core of polymeric mixed micelles [42].

The hydrodynamic size of PF127/Tween 80 mixed nanomicelles was measured by the DLS analysis, and the result is shown in Fig. 3. The average hydrodynamic size of mixed micelles was measured to be 22.1 $\mathrm{nm}$ with acceptable value of polydispersity index (PDI) of 0.2 , indicating a narrow size distribution of prepared nanomicelles. The surface charge of mixed nanomicelles, measured by zeta potential analysis, was $-7.63 \pm 0.56 \mathrm{mV}$, which is in the range of surface charge of pluronic micelles [17].

The morphology, size, and surface charge affect the ability of polymeric nanoparticles for internalization into cancer cells $[43,44]$. The mechanisms of cellular internalization nanoparticles include merging with cellular membranes or uptake by endocytosis [45-47]. The preferential accumulation of nanoparticles in tumor tissues has been known as the enhanced permeability and retention (EPR) effect [48]. Nanoparticles in the range of 10-100 nm have minimum accumulation in the liver and spleen which results in extended half-life in the blood and subsequently more accumulation in tumor tissues by EPR effect [49]. As can be observed, the size of mixed nanomicelles obtained in this study is in the optimum range of 10-100 nm which is suitable for efficient drug delivery to solid tumors [29, 48-50].

Figure 4 shows the percent cumulative release (\%Q) of quercetin from PF127/Tween 80 mixed nanomicelles compared to free drug at PBS solution ( $\mathrm{pH} \mathrm{7.4).} \mathrm{As} \mathrm{can} \mathrm{be} \mathrm{observed,} \mathrm{the} \mathrm{release} \mathrm{of} \mathrm{free} \mathrm{drug}$ was completed within $12 \mathrm{~h}$, whereas, a slow and gradual release of quercetin from the mixed nanomicelles was observed over a period of $120 \mathrm{~h}$ with cumulative drug release of $83.6 \%$. The sustained release of quercetin from mixed nanomicelles indicated its applicability as a controlled drug delivery system.

The anticancer activity of free quercetin, and quercetin-loaded PF127/Tween 80 mixed nanomicelles was evaluated by MTT assay using MCF7 cancer cells and the results are shown in Fig. 5 . The untreated cells and empty mixed micelles were kept as control and blank, respectively. The results revealed that the cytotoxicity of quercetin against MCF7 cells is significantly increased by encapsulation in nanomicelles (Fig. 5a). The IC50 concentrations of free quercetin and quercetin-loaded mixed nanomicelles were 31.2 $\mu \mathrm{g} / \mathrm{mL}$ and $14.1 \mu \mathrm{g} / \mathrm{mL}$, respectively. The lower cytotoxicity of free quercetin compared to its nanomicelles could be related to the aggregation of free drug in the cell culture medium which could prevent its adsorption and then passage into cancer cells. On the other hand, P-glycoproteins inhibition or efflux pump inhibitory activity of pluronic micelles may be responsible for increasing the cytotoxicity of encapsulated drug in pluronic micelles [51,52]. Moreover, the cytotoxicity of empty nanomicelles was evaluated and the results are shown in Fig. 5b. As can be observed, the empty mixed nanomicelles are safe (cell viability more than $90 \%$ ) even at high concentrations up to $2 \mathrm{mg} / \mathrm{mL}$ (equivalent to quercetinloaded mixed PF127/Tween 80 nanomicelles containing $50 \mu \mathrm{g} / \mathrm{mL}$ quercetin). This indicates that the empty nanomicelles have low toxicity and can be used as a safe drug carrier. Generally, the obtained results may provide an evidence for the potential clinical application of the prepared nanomicelles for cancer therapy. 


\section{Conclusions}

In this study quercetin-loaded mixed Pluronic F127 (PF127)/Tween 80 nanomicelles was prepared and their therapeutic potential against breast cancer cells was evaluated. The drug loading and entrapment efficiency of optimized nanomicelles were $2.3 \%$ and $98.0 \%$, respectively. The encapsulation of quercetin in mixed nanomicelles was confirmed by XRD analysis and FTIR spectroscopy. The mean hydrodynamic size and surface charge of mixed nanomicelles, measured by DLS and zeta potential analyses, were 22.1 $\mathrm{m}$ and $-7.63 \mathrm{mV}$, respectively. Moreover, in vitro cytotoxicity analyses on breast cancer cells (MCF7) showed that the encapsulation of quercetin in mixed nanomicelles can enhance cytotoxicity of drug, so that, quercetin-loaded mixed nanomicelles showed lower $\mathrm{IC}_{50}$ value $(8.9 \mu \mathrm{g} / \mathrm{mL})$ compared to free drug $(49.2 \mu \mathrm{g} / \mathrm{mL})$. Our results suggest that quercetin-loaded mixed nanomicelles have great potential to be used as a passive-targeted drug delivery system for breast cancer therapy.

\section{Declarations}

\section{Acknowledgement}

The authors gratefully acknowledge Arak University for the financial support of this research (Grant No. 97/13975).

\section{Conflicts of interest}

The authors declare no conflict of interest.

\section{References}

1. M. Bule, A. Abdurahman, S. Nikfar, M. Abdollahi, M. Amini, Food and chemical toxicology (2019)

2. N. Polerà, M. Badolato, F. Perri, G. Carullo, F. Aiello, Curr. Med. Chem. 26(31), 5825-5848 (2019)

3. A.V.A. David, R. Arulmoli, S. Parasuraman, Pharmacogn. Rev. 10(20), 84 (2016)

4. S. Wang, J. Yao, B. Zhou, J. Yang, M.T. Chaudry, M. Wang, F. Xiao, Y. Li, W. Yin, J. Food Prot. 81(1), 68-78 (2018)

5. S. Miltonprabu, M. Tomczyk, K. Skalicka-Woźniak, L. Rastrelli, M. Daglia, S.F. Nabavi, S.M. Alavian, S.M. Nabavi, Food Chem. Toxicol. 108, 365-374 (2017)

6. L. Wu, J. Li, T. Liu, S. Li, J. Feng, Q. Yu, J. Zhang, J. Chen, Y. Zhou, J. Ji, Cancer medicine 8(10), 4806-4820 (2019)

7. N. Kasiri, M. Rahmati, L. Ahmadi, N. Eskandari, H. Motedayyen, Inflammopharmacology, 1-24 (2019)

8. J.-H. Chang, S.-L. Lai, W.-S. Chen, W.-Y. Hung, J.-M. Chow, M. Hsiao, W.-J. Lee, M.-H. Chien, Biochimica et Biophysica Acta (BBA)-Molecular Cell Research 1864(10), 1746-1758 (2017)

9. A.B. Ward, H. Mir, N. Kapur, D.N. Gales, P.P. Carriere, S. Singh, World J. Surg. Oncol. 16(1), 108 (2018)

10. S. Hassanzadeh, Z. Feng, T. Pettersson, M. Hakkarainen, Polymer 74, 193-204 (2015) 
11. X. Zhang, J. Huang, C. Yu, L. Xiang, L. Li, D. Shi, F. Lin, OncoTargets and therapy 13, 513 (2020)

12. J. Sobczyński and B. Chudzik-Rząd, Design and Development of New Nanocarriers (Elsevier, 2018), pp. 331-364

13. W. Xu, P. Ling, T. Zhang, Journal of drug delivery 2013 (2013)

14. Y.H. Hussein, M. Youssry, Materials 11(5), 688 (2018)

15. X. Yuan, W. Ji, S. Chen, Y. Bao, S. Tan, S. Lu, K. Wu, Q. Chu, Int. J. Nanomed. 11, 2119 (2016)

16. S. Peng, W.-L. Hung, Y.-S. Peng, I.-M. Chu, J. Biomater. Sci. Polym. Ed. 25(12), 1225-1239 (2014)

17. C. Gorinova, D. Aluani, Y. Yordanov, M. Kondeva-Burdina, V. Tzankova, C. Popova, K. Yoncheva, Biotechnology \& Biotechnological Equipment 30(5), 991-997 (2016)

18. Y. Chen, W. Zhang, J. Gu, Q. Ren, Z. Fan, W. Zhong, X. Fang, X. Sha, International journal of pharmaceutics 452(1-2), 421-433 (2013)

19. Y. Chen, W. Zhang, Y. Huang, F. Gao, X. Sha, X. Fang, International journal of pharmaceutics 488(1-2), 44-58 (2015)

20. S. Di-Wen, G.-Z. Pan, L. Hao, J. Zhang, Q.-Z. Xue, P. Wang, Q.-Z. Yuan, International journal of pharmaceutics 500(1-2), 54-61 (2016)

21. R. Basak, R. Bandyopadhyay, Langmuir 29(13), 4350-4356 (2013)

22. E.V. Batrakova, A.V. Kabanov, Journal of controlled release 130(2), 98-106 (2008)

23. S. Park, H.S. Kim, W.J. Kim, H.S. Yoo, International journal of pharmaceutics 424(1-2), 107-114 (2012)

24. Y. Wang, L. Yu, L. Han, X. Sha, X. Fang, International journal of pharmaceutics 337(1-2), 63-73 (2007)

25. A.V. Kabanov, E.V. Batrakova, V.Y. Alakhov, Journal of controlled release 82(2-3), 189-212 (2002)

26. D.A. Chiappetta, A. Sosnik, Eur. J. Pharm. Biopharm. 66(3), 303-317 (2007)

27. A. Pitto-Barry, N.P. Barry, Polymer Chemistry 5(10), 3291-3297 (2014)

28. S. Shaarani, S.S. Hamid, N.H.M. Kaus, Pharmacognosy research 9(1), 12 (2017)

29. S. Biswas, P. Kumari, P.M. Lakhani, B. Ghosh, Eur. J. Pharm. Sci. 83, 184-202 (2016)

30. A.M. Butt, M.C.I.M. Amin, H. Katas, N. Sarisuta, W. Witoonsaridsilp, R. Benjakul, Journal of Nanomaterials 2012 (2012)

31. Y. Duan, X. Cai, H. Du, G. Zhai, Colloids Surf., B 128, 322-330 (2015)

32. L. Zhao, J. Du, Y. Duan, H. Zhang, C. Yang, F. Cao, G. Zhai, Colloids Surf., B 97, 101-108 (2012)

33. A. Parmar, S. Chavda, P. Bahadur, Colloids Surf., A 441, 389-397 (2014)

34. R. Mondal, N. Ghosh, S. Mukherjee, J. Phys. Chem. B 120(11), 2968-2976 (2016)

35. J. Bhattacharjee, G. Verma, V. Aswal, A.A. Date, M.S. Nagarsenker, P. Hassan, J. Phys. Chem. B 114(49), 16414-16421 (2010)

36. Z. Liu, D. Liu, L. Wang, J. Zhang, N. Zhang, Int. J. Mol. Sci. 12(3), 1684-1696 (2011) 
37. A. Pawar, S. Singh, S. Rajalakshmi, K. Shaikh, C. Bothiraja, Artificial cells, nanomedicine, and biotechnology 46(sup1), 347-361 (2018)

38. M. Asgari, M. Soleymani, T. Miri, A. Barati, J. Mol. Liq. 292, 111367 (2019)

39. J.W. Valle, A. Armstrong, C. Newman, V. Alakhov, G. Pietrzynski, J. Brewer, S. Campbell, P. Corrie, E.K. Rowinsky, M. Ranson, Investig. New Drugs 29(5), 1029-1037 (2011)

40. M. Catauro, F. Papale, F. Bollino, S. Piccolella, S. Marciano, P. Nocera, S. Pacifico, Sci. Technol. Adv. Mater. 16(3), 035001 (2015)

41. H.-J. Li, A.-Q. Zhang, Y. Hu, L. Sui, D.-J. Qian, M. Chen, Nanoscale Res. Lett. 7(1), 612 (2012)

42. B.M. Davis, M. Pahlitzsch, L. Guo, S. Balendra, P. Shah, N. Ravindran, G. Malaguarnera, C. Sisa, E. Shamsher, H. Hamze, Scientific reports 8(1), 1-13 (2018)

43. H. Lee, B. Hoang, H. Fonge, R.M. Reilly, C. Allen, Pharmaceutical research 27(11), 2343-2355 (2010)

44. H. Cabral, Y. Matsumoto, K. Mizuno, Q. Chen, M. Murakami, M. Kimura, Y. Terada, M. Kano, K. Miyazono, M. Uesaka, Nature nanotechnology 6(12), 815 (2011)

45. V.P. Torchilin, Nanotechnology in Drug Delivery (Springer, 2009), pp. 313-346

46. R.W. Horobin, Organelle-specific pharmaceutical nanotechnology 2010, 193-206 (2010)

47. G. Sahay, D.Y. Alakhova, A.V. Kabanov, Journal of controlled release 145(3), 182-195 (2010)

48. K. Greish, Cancer nanotechnology (Springer, 2010), pp. 25-37

49. S.N. Ekdawi, A.S. Mikhail, S. Stapleton, J. Zheng, S. Eetezadi, D.A. Jaffray, C. Allen, Cancer Targeted Drug Delivery (Springer, 2013), pp. 543-571

50. N. Hoshyar, S. Gray, H. Han, G. Bao, Nanomedicine 11(6), 673-692 (2016)

51. H. Maeda, Advances in enzyme regulation (2001)

52. S. Croy, G. Kwon, Curr. Pharm. Design 12(36), 4669-4684 (2006)

\section{Figures}




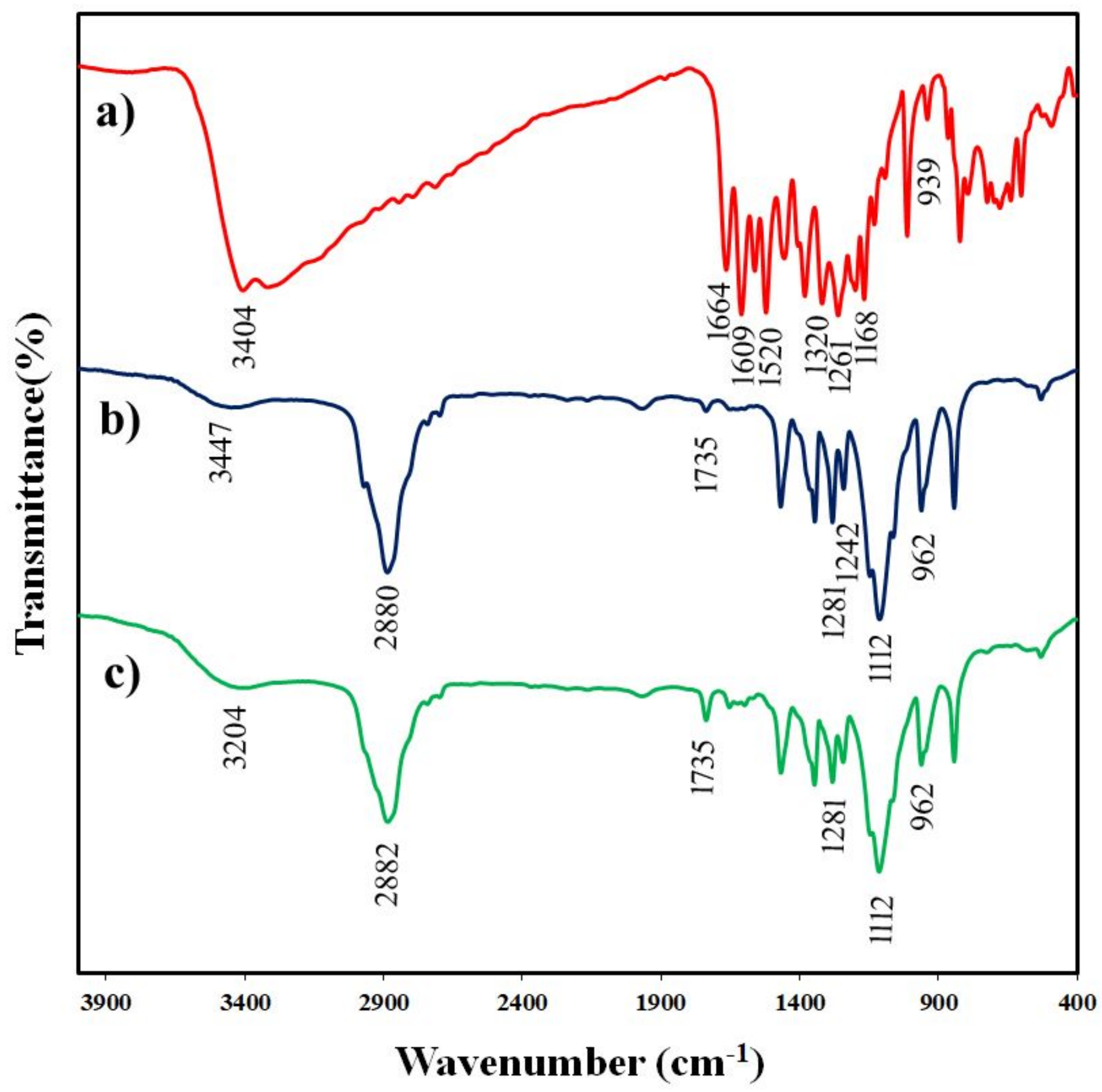

Figure 1

FTIR spectra of a) pure quercetin, b) empty nanomicelles, and c) quercetin-loaded mixed nanomicelles 


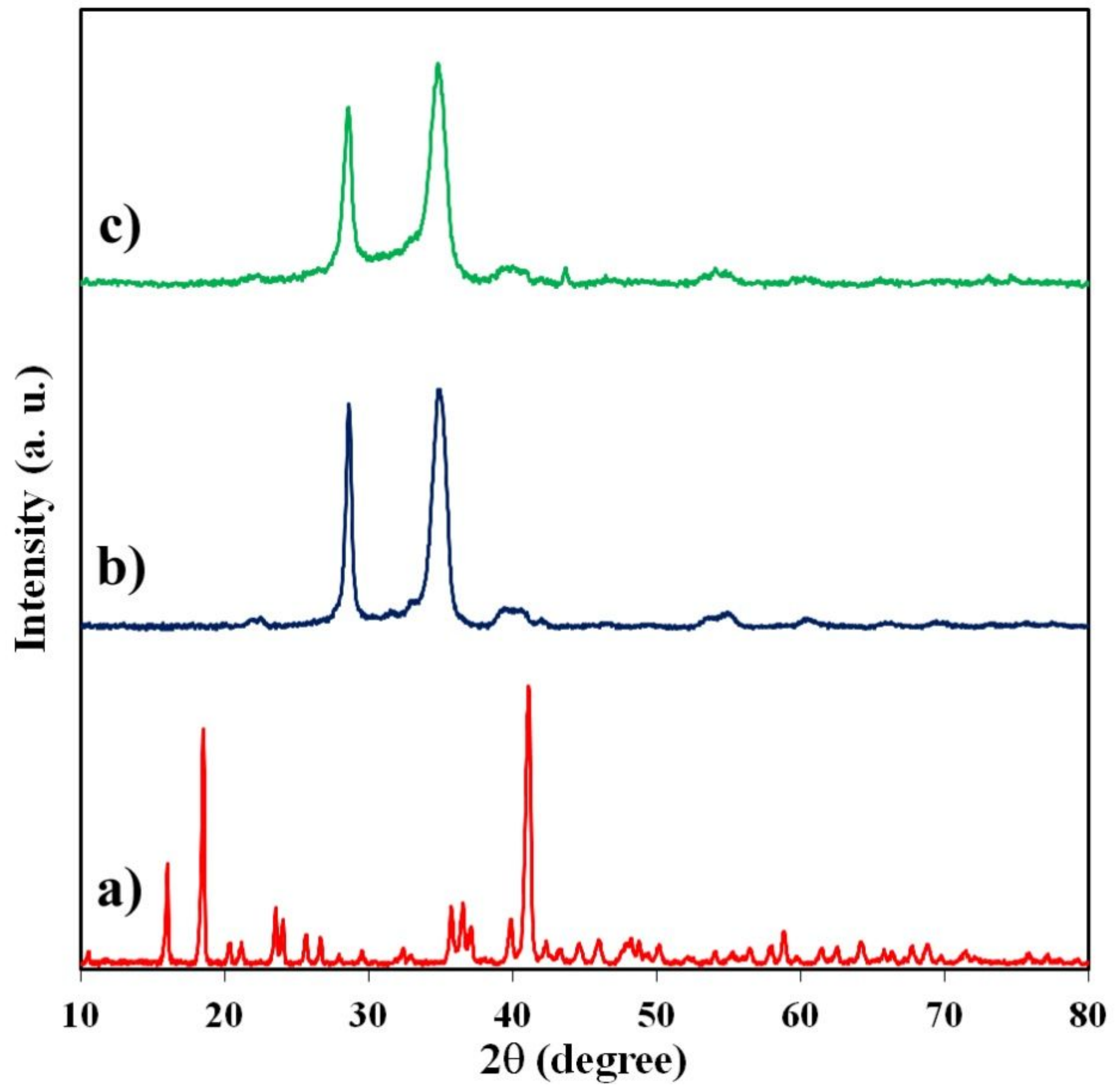

Figure 2

XRD patterns of a) pure quercetin, b) empty nanomicelles, and c) quercetin-loaded mixed nanomicelles 


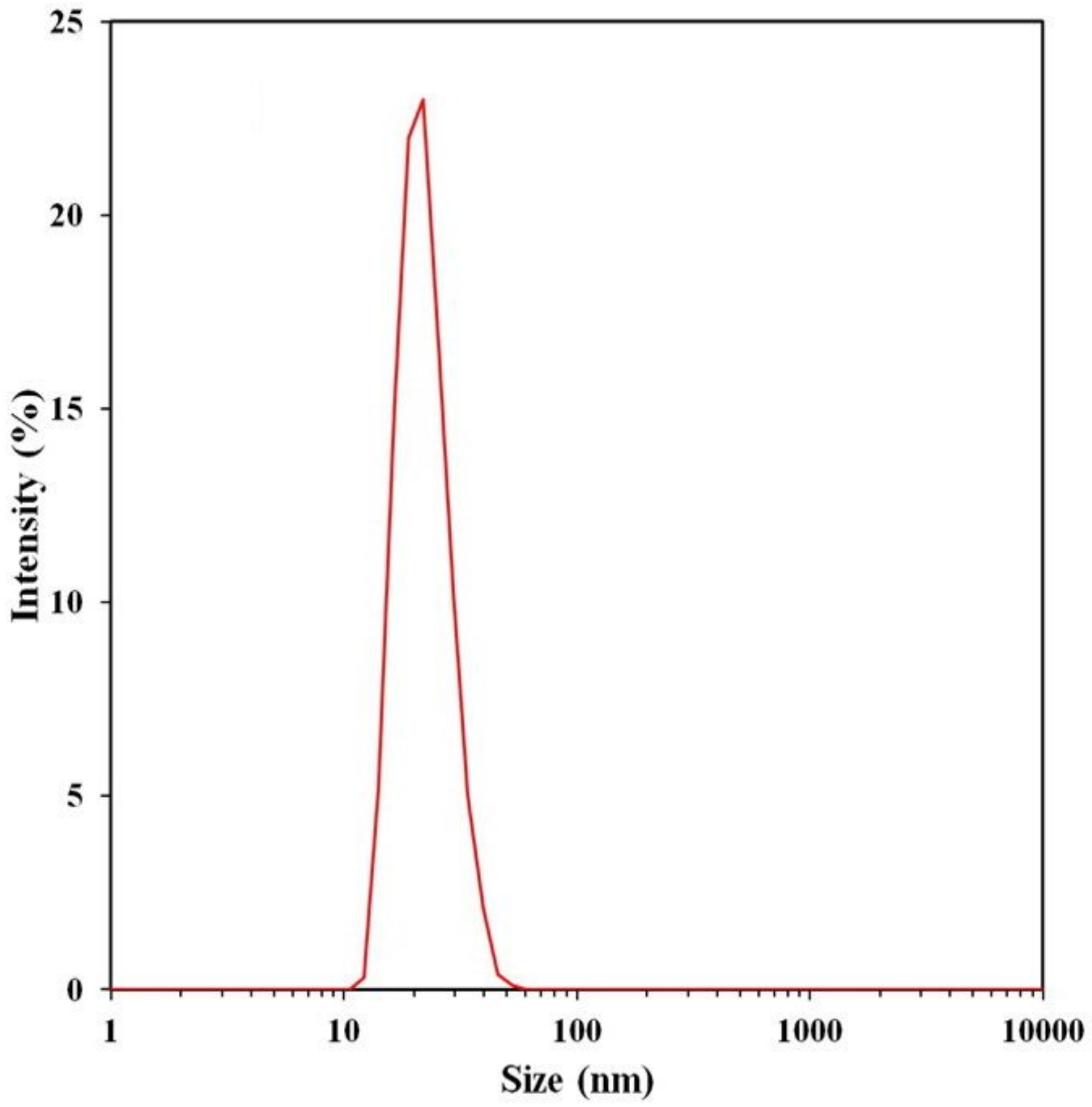

Figure 3

hydrodynamic size distribution of the prepared nanomicelles 


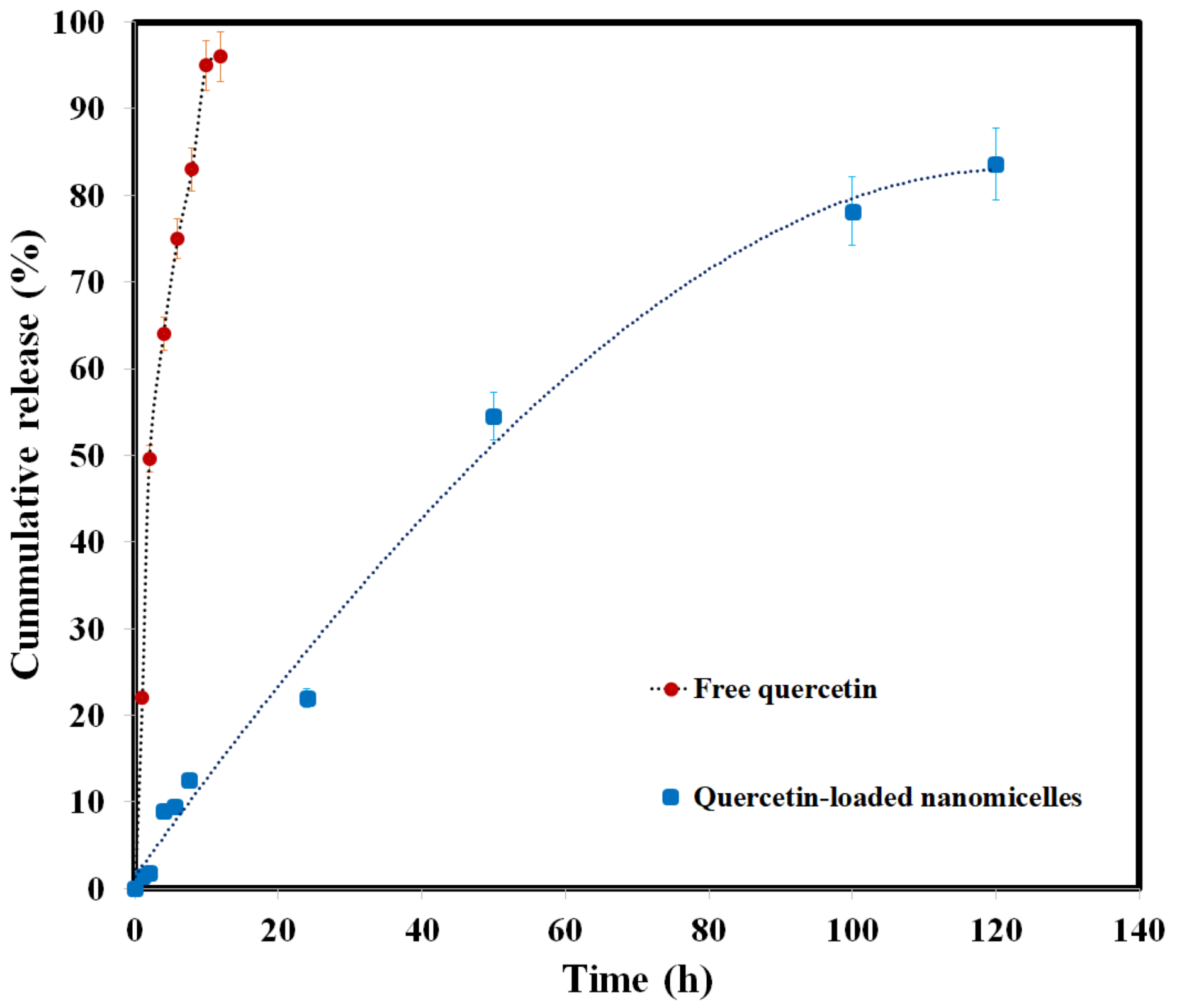

Figure 4

Cumulative release of quercetin from PF127/Tween 80 mixed nanomicelles and as free drug 

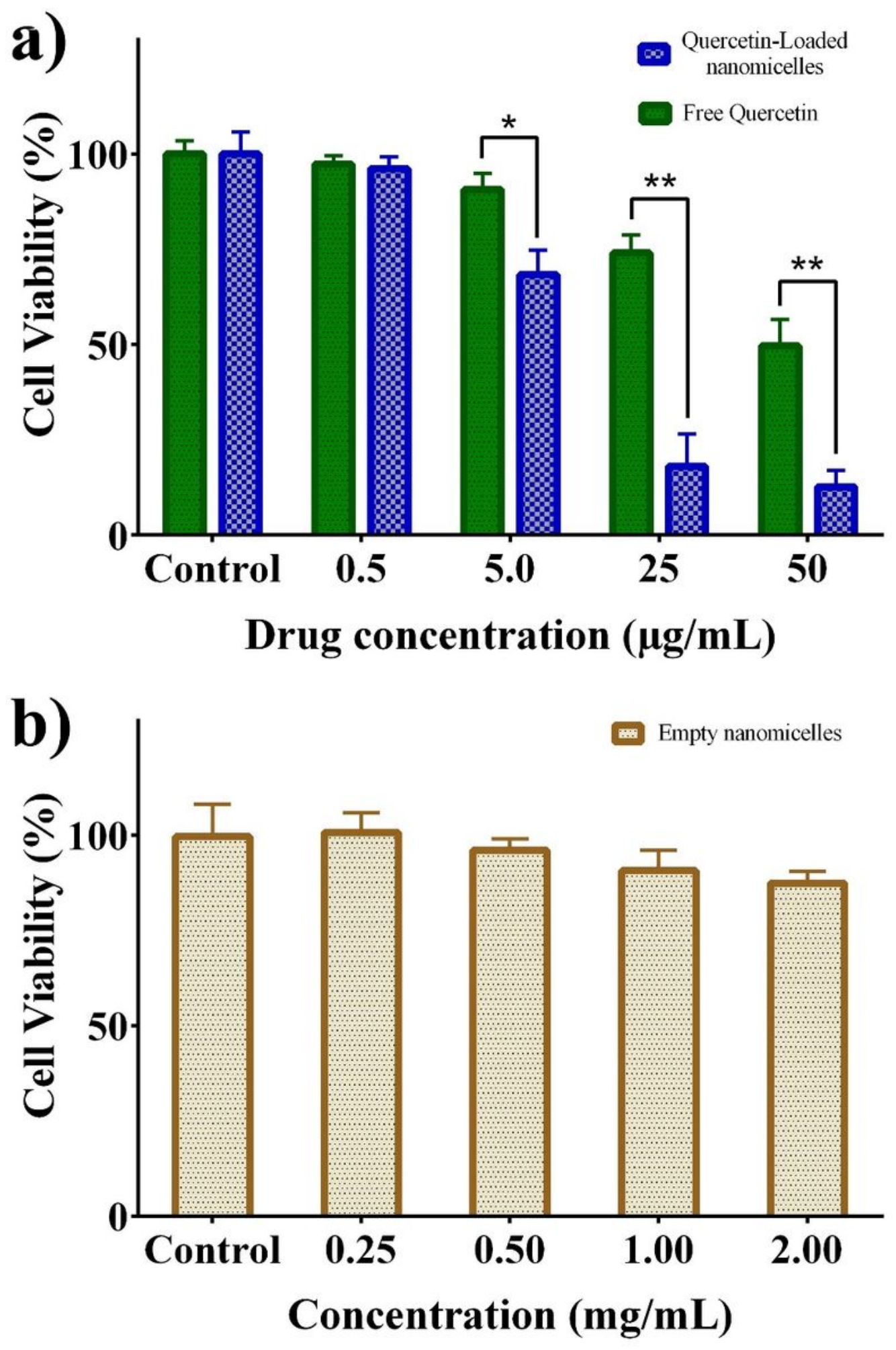

Figure 5

Cytotoxicity of free quercetin, and quercetin-loaded mixed nanomicelles against MCF7 cancer cells after $48 \mathrm{~h}$ incubation, b) The viability of MCF7 cells exposed for $48 \mathrm{~h}$ to empty mixed nanomicelles as that used to for encapsulation of quercetin by mixed nanomicelles, $(*)$ indicates Pvalue $<0.05$, and $(* \star)$ indicates Pvalue $<0.005$ 